\title{
MEDICIÓN DE LOS PROGRESOS Y LA EVOLUCIÓN DE LOS AGRONEGOCIOS: UN ANÁLISIS BIBLIOMÉTRICO DE LA LITERATURA DE 2001 A 2020
}

\section{MEASURING PROGRESS AND EVOLUTION OF AGRIBUSINESS: A BIBLIOMETRIC ANALYSIS OF THE LITERATURE FROM 2001 TO 2020.}

Laura Carolina Nadir Romero Vargas (Universidad Autónoma de Zacatecas) ${ }^{1}$

Soraida Natali Suárez Torres (Universidad Autónoma de Zacatecas) ${ }^{2}$

Ildefonso Narvaez Ortiz (Universidad Autónoma Chapingo) ${ }^{3}$

\section{Resumen:}

En los últimos años, los agronegocios han evolucionado de una posición meramente productiva a una de mayor valor añadido. La evolución de los agronegocios ha venido propiciada principalmente por la interacción de factores económicos, políticos, sociales y tecnológicos, principalmente. En este sentido, la nueva figura de los agronegocios trae consigo cambios en los mercados, sistemas productivos y relaciones entre agentes, priorizando la innovación, sostenibilidad y el desarrollo desde un enfoque local y global. Sin embargo, la evolución de los agronegocios dificulta la identificación y el entendimiento de sus principales áreas de investigación y desarrollo. En este sentido, la presente investigación permite identificar, visualizar y analizar las principales líneas de investigación y desarrollo del campo de agronegocios a través del uso de técnicas y herramientas bibliométricas. Para ello, se han analizado todas las publicaciones relacionadas con agronegocios disponibles en la base de datos Scopus entre 2001 y 2020. Este análisis evalúa la productividad e impacto de los principales autores, las organizaciones y países más productivos, y las principales líneas de investigación y sus componentes, estableciendo un marco de referencia para otras investigaciones.

Palabras Clave: Agronegocios, Sustentabilidad, Economía Agrícola, Mapas de la Ciencia, Análisis Bibliométrico.

Códigos JEL: Q00, Q01.

\footnotetext{
Abstract:

${ }^{1}$ lauracnrvargas@gmail.com, Universidad Autónoma de Zacatecas.

2 nata.li.1515@hotmail.com, Universidad Autónoma de Zacatecas.

3 ildenar@gmail.com, Universidad Autónoma Chapingo.

Recibido: 17 de abril de 2020. Aceptado: 15 de junio de 2020.
}

In recent years, agribusinesses have evolved from a purely productive position to one of greater added value. The evolution of agribusinesses has been driven mainly by the interaction of economic, political, social and technological factors. In this respect, the new figure of agribusinesses entails changes in markets, production systems and relations between stakeholders, prioritizing innovation, sustainability and development from a local and global approach. However, the evolution of agribusinesses hinders the identification and understanding of its main areas of research and development. In this 
sense, this research allows the identification, visualization and analysis of the main areas of research and development in the field of agribusiness by the use of bibliometric techniques and tools. To this purpose, all publications related to agribusinesses available in the database Scopus between 2001 and 2020 have been analyzed. This analysis evaluates the productivity and impact of the main authors, the most productive organizations and countries, and the main themes and their components, establishing a reference framework for other research.

Key Words: Agribusiness, Sustainability, Agricultural Economics, Science Map, Bibliometric Analysis.

JEL Codes: Q00, Q01.

\section{INTRODUCCIÓN}

Los agronegocios, se entienden como actividades económicas implementadas en el medio rural, que guardan relación con el uso y aprovechamiento de los recursos naturales agropecuarios y forestales, para el desarrollo de productos específicos orientados a las necesidades del consumidor final, por medio de la producción primaria, procesamiento, transformación, almacenamiento, distribución y comercialización, generando utilidades económicas en forma de ganancia para aquellos que emprenden estas actividades (Gómez et al., 2008; Rodríguez, 2010).

El auge de los agronegocios va de la mano con los cambios en los mecanismos del uso de la tierra y de las actividades agrícolas, para afrontar y dar soluciones a problemas de nivel global, y aportar al cumplimiento de los Objetivos de Desarrollo Sostenible (ODS) para el 2030. De igual manera, estos objetivos, que están relacionados, señalan que el uso de un área influye en los resultados de otras, por lo que el desarrollo debe estar en equilibrio, con la sostenibilidad del medio ambiente, económico y social (CEPAL, 2018). Los retos a los que se enfrenta este sector se relacionan con los cambios de prácticas agrícolas, aplicación de alianzas estratégicas tanto con instituciones, gremios y/o productores para lograr ser más competitivos, y la capacidad para posicionar determinados productos o servicios en el mercado, donde existe oportunidades de ganancia y expansión bajo ciertos parámetros, generando beneficios de manera responsable, no solo para la empresa, sino para todos los agentes involucrados (Rojas y Sepúlveda, 1999).

América Latina se ha caracterizado por ser el mayor oferente de materias primas, y sus transformaciones en áreas rurales, han dado como resultado un alto crecimiento y desarrollo a nivel global (Teubal, 2002; Ceroni, 2017). Un factor importante, para ampliar el concepto de agronegocio, es la integración de actividades económicas como servicios ambientales, comercio, turismo ecológico, certificaciones, mercado internacional, entre otros. (Castellano, 2013). El crecimiento de los agronegocios influye en la diferenciación de sus productos en nichos y segmentos específicos, por lo que este sector adquiere oportunidades, ya que se promueve la integración de una sociedad más justa y equitativa, en el que el escenario de los agronegocios aporta a la estabilidad social, el crecimiento económico y la sostenibilidad de los recursos naturales (CEPAL, 2018).

Tomando en cuenta esta situación, el objetivo principal de este estudio es retratar el presente de la investigación en agronegocios en Latinoamérica en el periodo de 2001 al 2020. Para ello, se ha desarrollado un análisis bibliométrico, que busca cuantificar la actividad científica por medio de indicadores bibliométricos, que permiten entender y 
describir la influencia en el entorno (Pritchard, 1996; Bordons, 2002). Además, para un mejor entendimiento de los resultados se recurrió a la confección de los mapas científicos de este campo de estudio, herramienta utilizada para el estudio de la estructura y dinamismo de un campo científico determinado, ya que permiten representar de forma dinámica los resultados obtenidos (Buter et al., 2006; Van Eck y Walmant, 2010).

Por último, la información que se presenta a continuación, se divide de la siguiente manera: (i) se hace una descripción de la evolución del concepto de agronegocios; (ii), se muestra la metodología implementada para el análisis bibliométrico con base en los artículos recopilados; (iii), se presenta la evaluación de rendimiento bibliométrico, mapas científicos, y los artículos, fuentes de información, y autores más productivos respecto a la consulta realizada, y (iv), se presentan las principales conclusiones.

\section{EVOLUCIÓN DE LOS AGRONEGOCIOS}

El término de agronegocio implica más que la interrelación con otros sectores, propios de los sistemas involucrados en la optimización de la agroindustria. Este término se enfoca en nuevas formas de gestión y crecimiento de esos vínculos, recursos y factores incluidos, para un mejor desarrollo de mecanismos de integración en la red de valor de los productos y servicios (Gras y Hernández, 2013). El término agronegocio se enfoca en la sinergia empresarial que relaciona distintos sectores de la cadena agroindustrial, formando áreas que generan nuevas tendencias de gestión por parte de los actores involucrados.

Los economistas John Davis y Ray Goldberg, a quienes se les conoce como los padres del marco teórico del concepto de agronegocios en el año 1957, lo definieron de esta forma: "La suma del total de operaciones involucradas en la manufactura y en la distribución de la producción agrícola; operaciones de la producción en el campo, en el almacenaje, el procesamiento, y distribución de los commodities agrícolas y las manufacturas hechas con los mismos” (Davis y Goldberg, 1957).

Esta definición evoluciona a un concepto más amplio y dinámico, en el cual su posición central no está en las operaciones, sino en las personas que realizan las operaciones. Este sistema de agronegocios involucra toda la red de valor para la producción, procesamiento y comercialización, por lo tanto, reúne todo los actores o participantes de los eslabones de estas áreas de un producto o servicio agropecuario. Este sistema considera el área cultivada, procesos productivos, colaboradores, intermediarios, canales de distribución y consumidor final, en un determinado flujo de información para una adecuada coordinación de planificación, etapas y objetivos. También se incluye las instituciones que afectan o favorezcan las fases de flujo, como gobiernos, mercados futuros, y asociaciones comerciales (Ray Goldberg, 1968). Es una definición en la que su foco son todos los actores, factores y eslabones que comprenden la actividad del flujo de agronegocios.

En los años cincuenta, se desarrollaron nuevos estudios de transformaciones enfatizadas en el funcionamiento y utilidad de las unidades agropecuarias, generando nuevos modelos representativos, insertando la agricultura en el auge del funcionamiento del sistema capitalista. La contribución que se generó de los principales autores sobre agronegocios, promovió una serie de transformaciones que se desarrollaron en la red de valor de los procesos productivos agropecuarios, bajo un flujo de información directo entre productor y consumidor final, promoviendo el desarrollo de empresas y su posicionamiento en el mercado. (Posada et al., 1996). 
Históricamente como ha ocurrido en muchas etapas de crisis económicas y sociales en el mundo, se buscan las estrategias que generen un nuevo auge para el crecimiento del capital, por lo que se empiezan a desarrollar políticas de manera estructural en un hito determinado. En los agronegocios, esta etapa fue en la posguerra, por lo que lo que las consecuencias obtenidas en este factor, como la tecnología, favoreció para el crecimiento del sector productivo, aumentando oportunidades de mercado, organización y negocio (Perkins, 1997; Ceroni, 2018).

En este sentido, América Latina ha incursionado en la segmentación internacional del trabajo como proveedora de materias primas, por lo que ha desarrollado un factor diferencial en el mercado mundial (Ceroni, 2017). El sector de los agronegocios ha desarrollado una transformación a nivel global por la inclusión de nuevas tecnologías que han permitido la automatización de diversas actividades en este sector. Sin embargo, en muchos países en vía de desarrollo no se tienen las condiciones necesarias para desarrollar innovación tecnológica y propiedad intelectual (Cáceres, 2015).

Para integrar este cambio en los procesos productivos, es necesario desmitificar aspectos como que la tecnología son herramientas innecesarias para los pequeños productores, o que por sus costos no son recomendables, o que las empresas dedicadas a los agronegocios han de ser flexibles para enfrentar los cambios del mercado y ser competitivas en su entorno. Se tiene que entender que la tecnología es un sistema de gestión integral en la organización, para la utilización efectiva de los recursos, creando así valor en los productos que se lanzan en el mercado, incluso si se trata de una producción a pequeña escala (Cerón, Mario y Barrios, Dursun. 2019). En este sentido, las sinergias entre empresas, que buscan el desarrollo de nuevas tecnologías en las que la innovación sea un pilar importante del modelo de agronegocio son cada vez mayores, generando así alianzas, cooperativas estratégicas, y redes de valor más estructurales, orientadas al desarrollo de productos acordes a las necesidades del consumidor final.

El avance en términos de innovación y tecnología, que se ha presentado en el contexto de agronegocio, es imprescindible para su fomento y planificación estratégica, cuyo propósito es relacionar los insumos con el capital, optimizando los factores que permitan aumentar la eficiencia en la productividad (Ceroni, 2017). Las nuevas tendencias tecnológicas en este sector han incrementado de manera significante la productividad de la mano de obra rural (Martínez Castillo, 2010), mejorando la calidad del trabajo. De esta manera los resultados se ven reflejados en el incremento de la rentabilidad, la producción y el posicionamiento en los mercados a nivel global (Pingali, 2012). De este modo busca la relación eficaz entre las cadenas, minimizando las unidades de tiempo y optimizando la producción (Leff, 1994).

La aplicación de la tecnología en las etapas de las operaciones de los agronegocios, ha generado debates. Hay quienes destacan su potencial productivo, su capacidad para producir comida para una población cada vez más numerosa, y sus impactos positivos en la optimización de tiempos y reducción de costos (Borlaug, 2000), aunque otros marcan sus impactos negativos en el entorno ambiental, social y económico (Patel, 2012).

Por lo tanto, cuando se trata de procesos relacionados con los agronegocios, es necesario entenderlos como algo que influye en el crecimiento agrícola y el aumento de la productividad, según el sistema implementado para el proceso productivo (Heredia et al. 2010). Es un sistema que opera bajo las demandas de los mercados y a las características, circunstancias y requerimientos de los consumidores, con una planificación estratégica que vincula redes de valor, innovación, y tecnología, generando sostenibilidad en cada proceso desarrollado y sinergias para la creación de valor de productos diferenciales, para alcanzar así la competitividad en nuevos mercados. 


\section{METODOLOGÍA}

Tomando en cuenta lo anterior, se definió una consulta para recopilar los documentos relacionados con agronegocios disponibles entre los años 2001 y 2020 en la base de datos de Scopus a 3 de marzo de 2020. Para ello, se utilizó la siguiente búsqueda avanzada que concentra ocho palabras clave vinculadas a través de operadores booleanos: TITLE-ABS-KEY ("agrobusiness" OR “agro-business" OR "agro business”) OR TITLE-ABS-KEY (“agribusiness" OR “agri-business” OR "agri business”) OR TITLEABS-KEY ("agronegocios” OR "agronegocio").

El análisis bibliométrico se hizo con base a la cantidad de publicaciones, cantidad de citas, h-index e índice SJR y como resultado se obtiene la clasificación de autores, artículos y fuentes que permiten identificar los actores más representativos de la consulta de agronegocios en la base de Scopus (Hirsch, 2005; Silva, 2012).

Las bases de datos se prepararon en una hoja de cálculo y los mapas científicos fueron construidos con el programa VOSviewer, lo que permitió vincular las relaciones que hay entre palabras clave y los principales indicadores (Van Eck y Waltman, 2010; Guzman y Trujillo, 2013). Además, con el programa estadístico R (R Core Team, 2020) se construyeron las gráficas de líneas y barras, que fueron complementados con la gráfica de nube de palabras de autores utilizando el paquete wordcloud (Fellows, 2018).

Las líneas de investigación corresponden a las palabras claves que se repiten y vinculan en los artículos encontrados en la consulta, representan las bases y áreas en que se apoyan los agronegocios para estructurarse y expandirse. Se configuran como los temas de investigación representativos dentro de la literatura de la consulta (Callon et al., 1991; Miguel et al., 2008). En este sentido, los mapas científicos permiten la visualización de las principales relaciones entre las líneas de investigación y su intensidad.

Cada artículo se verifico individualmente, para corroborar su enfoque, y encaje con el ámbito estudiado, y así evitar resultados erróneos respecto a la información analizada. Es importante indicar que las consultas y los mapas científicos se han desarrollado utilizando los conceptos en inglés, ya que todos los documentos presentan título, resumen y palabras clave en este idioma.

Las siguientes secciones, se enfoca en la evaluación y análisis de los resultados obtenidos por los indicadores bibliométricos en las publicaciones, citas, distribución geográfica, fuentes de información, y autores; para identificar a los autores más productivos, y la relación entre los mismos, y determinar la influencia e impacto de estos en la literatura sobre agronegocios. De igual manera se presenta el análisis de los mapas científicos con las respectivas líneas de investigación, y por último se recopilan las conclusiones del estudio y la evolución del enfoque de los agronegocios.

\section{EVALUACIÓN}

En esta sección se muestran los resultados obtenidos de los documentos recuperados y sus variables utilizadas para el análisis bibliométrico, es decir autores, citas, áreas de investigación, distribución geográfica, y fuentes de información más productivas. De igual manera, sus correspondientes mapas científicos, donde se visualiza los temas de investigación más relevantes, la interrelación de los mismos, y evolución utilizando la herramienta VOSviewer (López-Robles et al., 2017).

Se obtuvieron 121 documentos que se componen de 110 artículos, 9 de revisión y 2 publicaciones de congresos. En la Figura 1, se presenta la distribución anual de las publicaciones y citas totales. En el periodo entre 2001 y 2005 la productividad es baja, 
del orden de una (1) publicación, que su vez, no fueron citadas en el mismo periodo. A partir del 2006 hay una tendencia creciente hasta el 2018 que alcanza 21 publicaciones, y donde se da una caída hasta 10 publicaciones en 2019, es importante notar que a pesar de la reducción en publicaciones la cantidad de citas sigue en aumento alcanzando 41 en 2019. El 2020 se incluye como una referencia de las publicaciones hasta la fecha de la consulta, mas no es comparable con el resto, por lo tanto, no se puede considerar como cambio de tendencia. Con base en la información disponible el año 2019 puede considerarse como un valor atípico de publicaciones y que se retome la tendencia creciente a partir de 2020. Finalmente, el periodo más productivo fue del 2015 a 2019 con un total de 71 publicaciones, y en el mismo periodo se acumularon 155 citas con tendencia creciente. Lo que permite inferir que en el ámbito académico y científico hay una progresiva acogida del concepto de agronegocios y su aplicación en el entorno latinoamericano.

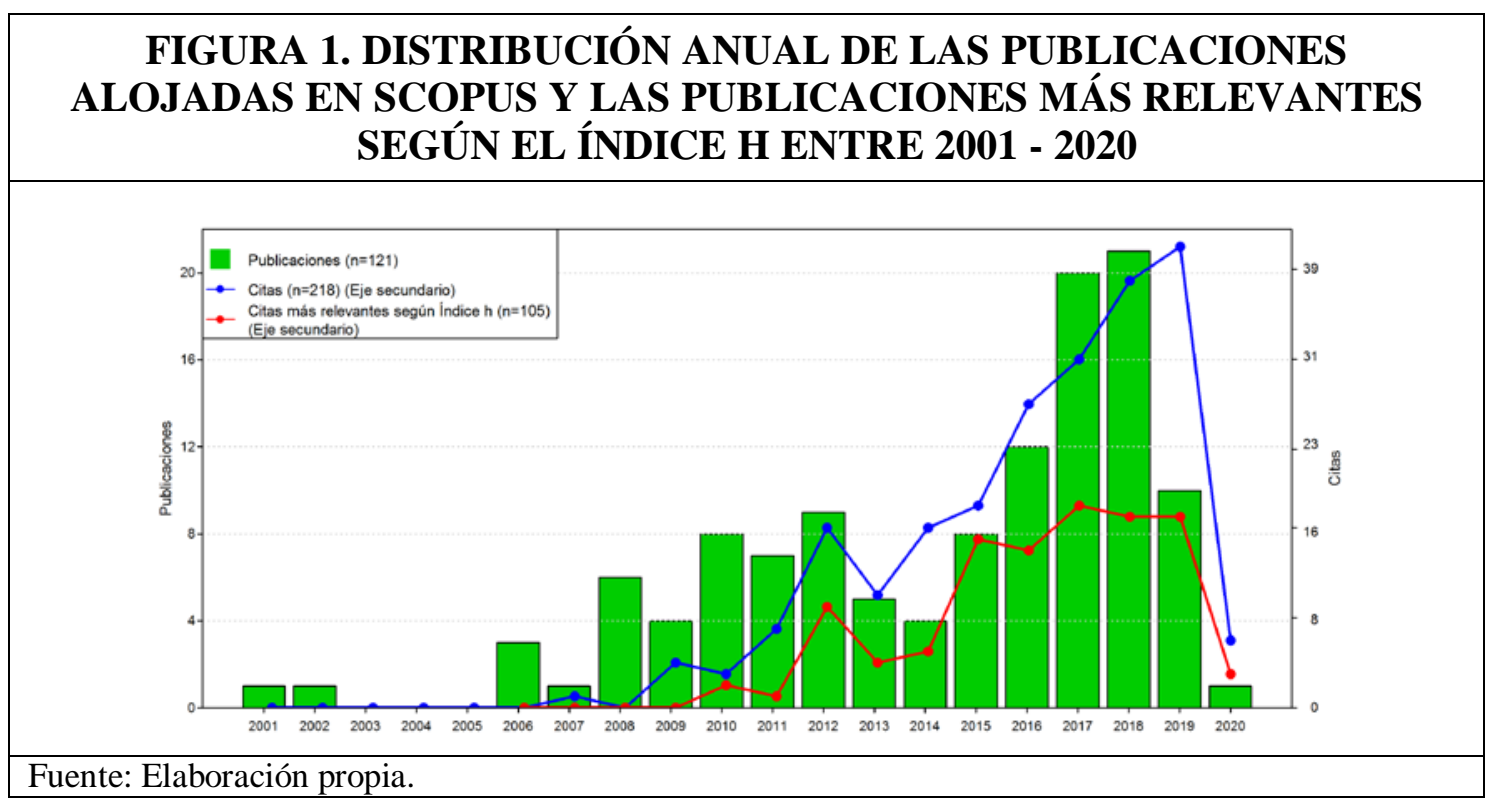

Las áreas de conocimiento que abarca los Agronegocios presentan un crecimiento, siendo las más representativas Business and International Management (24), Agricultural and Biological Sciences (miscellaneous) (14), Social Sciences (miscellaneous) (11) y las principales fuentes de información en su mismo orden son Espacios, Revista en Agronegocio e Meio Ambiente y Revista de Economía y Sociología Rural, tal como se puede observar en la Tabla 1, donde se identificó las principales fuente más productivas de los Agronegocios.

Los países más productivos son: Brasil (63) y Venezuela (29) (ver Figura 2). Además, son referentes en este estudio por su impacto de investigación en los agronegocios. La mayor parte de las publicaciones se registran en América Latina, esta región ha sido la principal oferente de insumos y productos agrícolas en el mundo; está actividad económica que se obtiene del trabajo del campo, no solo a la producción de los productos, sino de su procesamiento, transporte y comercialización. Este sector, no solo mejora la seguridad alimentaria, sino que resulta importante para el desarrollo económico de muchos países, en especial los subdesarrollados. 


\section{FIGURA 2. PAÍSES MÁS PRODUCTIVOS EN LOS AGRONEGOCIOS 2001 A 2020}

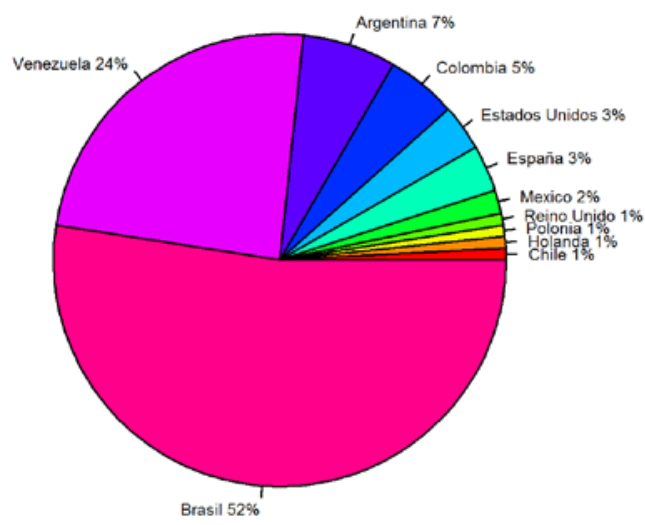

Fuente: Elaboración propia.

\section{TABLA 1. FUENTES MÁS PRODUCTIVAS EN EL ÁMBITO DE LOS AGRONEGOCIOS}

\begin{tabular}{|c|c|c|c|c|c|}
\hline Revista (País) & $\begin{array}{c}\text { SJR } \\
(2018)\end{array}$ & $\begin{array}{c}\text { Ámbito } \\
\text { (Mejor cuartil) }\end{array}$ & Publicaciones & $\begin{array}{l}\text { Publicaciones } \\
\text { (Índice h ) }\end{array}$ & $\begin{array}{c}\text { Citas } \\
\text { totales } \\
\text { (auto citas) } \\
\end{array}$ \\
\hline $\begin{array}{l}\text { Espacios } \\
\text { (Venezuela) }\end{array}$ & 0.158 & $\begin{array}{l}\text { Business and } \\
\text { International } \\
\text { Management (Q3) }\end{array}$ & 24 & 12 & $638(282)$ \\
\hline $\begin{array}{l}\text { Revista em } \\
\text { Agronegocio e } \\
\text { Meio Ambiente } \\
\text { (Brasil) }\end{array}$ & 0.11 & $\begin{array}{l}\text { Agricultural and } \\
\text { Biological Sciences } \\
\text { (miscellaneous) (Q4) }\end{array}$ & 14 & 5 & $16(0)$ \\
\hline $\begin{array}{l}\text { Revista de } \\
\text { Economia e } \\
\text { Sociologia Rural } \\
\text { (Brasil) }\end{array}$ & 0.279 & $\begin{array}{l}\text { Social Sciences } \\
\text { (miscellaneous) (Q2) }\end{array}$ & 11 & 13 & $37(8)$ \\
\hline $\begin{array}{l}\text { Mundo Agrario } \\
\text { (Argentina) }\end{array}$ & 0.149 & Urban Studies (Q3) & 6 & 7 & $18(3)$ \\
\hline $\begin{array}{l}\text { Agroalimentaria } \\
\text { (Venezuela) }\end{array}$ & 0.106 & $\begin{array}{l}\text { Economics, } \\
\text { Econometrics and } \\
\text { Finance } \\
\text { (miscellaneous) (Q4) }\end{array}$ & 5 & 6 & $3(0)$ \\
\hline $\begin{array}{l}\text { Latin American } \\
\text { Perspectives } \\
\text { (Estados Unidos) }\end{array}$ & 0.479 & $\begin{array}{l}\text { Geography, Planning } \\
\text { and Development } \\
\text { (Q2) }\end{array}$ & 3 & 34 & 167 (14) \\
\hline $\begin{array}{l}\text { Revista Brasileira } \\
\text { de Economia } \\
\text { (Brasil) }\end{array}$ & 0.155 & $\begin{array}{l}\text { Economics, } \\
\text { Econometrics and } \\
\text { Finance } \\
\text { (miscellaneous) (Q3) }\end{array}$ & 3 & 10 & $17(2)$ \\
\hline $\begin{array}{l}\text { Revista Brasileira } \\
\text { de Gestao e } \\
\text { Desenvolvimento } \\
\text { Regional (Brasil) }\end{array}$ & 0.162 & $\begin{array}{l}\text { Environmental } \\
\text { Science } \\
\text { (miscellaneous) (Q3) }\end{array}$ & 3 & 5 & $14(4)$ \\
\hline
\end{tabular}




\begin{tabular}{|c|c|c|c|c|c|}
\hline $\begin{array}{l}\text { Revista Brasileira } \\
\text { de Zootecnia } \\
\text { (Brasil) }\end{array}$ & 0.384 & $\begin{array}{l}\text { Animal Science and } \\
\text { Zoology (Q2) }\end{array}$ & 2 & 48 & $236(15)$ \\
\hline $\begin{array}{l}\text { Revista Brasileira } \\
\text { de Engenharia } \\
\text { Agricola e } \\
\text { Ambiental (Brasil) }\end{array}$ & 0.519 & $\begin{array}{l}\text { Agronomy and Crop } \\
\text { Science (Q2) }\end{array}$ & 2 & 27 & 459 (88) \\
\hline $\begin{array}{l}\text { Custos e } \\
\text { Agronegocio } \\
\text { (Brasil) }\end{array}$ & 0.209 & $\begin{array}{l}\text { Agronomy and Crop } \\
\text { Science (Q3) }\end{array}$ & 2 & 5 & 79 (38) \\
\hline Producao (Brasil) & 0.145 & $\begin{array}{l}\text { Industrial and } \\
\text { Manufacturing } \\
\text { Engineering (Q3) }\end{array}$ & 2 & 12 & $98(3)$ \\
\hline $\begin{array}{l}\text { Revista de } \\
\text { Sociologia e } \\
\text { Politica (Brasil) }\end{array}$ & 0.48 & $\begin{array}{l}\text { Sociology and } \\
\text { Political Science } \\
\text { (Q2) }\end{array}$ & 2 & 9 & $40(6)$ \\
\hline $\begin{array}{l}\text { Scripta Nova } \\
\text { (España) }\end{array}$ & 0.172 & $\begin{array}{l}\text { Geography, Planning } \\
\text { and Development } \\
\text { (Q3) }\end{array}$ & 2 & 7 & $29(0)$ \\
\hline
\end{tabular}

Fuente: Elaboración propia.

\section{AUTORES MÁS PRODUCTIVOS Y MÁS CITADOS}

En la tabla 2, se presenta los autores más productivos con base en la cantidad de publicaciones realizadas, el más representativo es Guilhoto, J.J.M y sus aportes se concentran en el ámbito empresarial y familiar, que además es economista de la Organización para la Cooperación y el Desarrollo Económicos (OCDE). Antes de unirse a la OCDE, fue profesor en el Departamento de Economía de la Universidad de São Paulo (USP), Brasil. La mayor parte de los autores reúnen dos publicaciones. En este sentido, en la Figura 3 se muestran en una nube los autores más citados en el ámbito de los agronegocios, el tamaño y los colores representan la cantidad de citas sobre los grupos que se forman; durante el periodo 2001 al 2020, y se identifica a Heredia B., Palmeira M. y Leite S.P entre los más citados, y se resalta a Guilhoto, J.J.M, ya que además de ser uno de los más citados también se encuentra clasificado como el de mayor productividad.

\begin{tabular}{|l|}
\hline \multicolumn{1}{|c|}{ TABLA 2. AUTORES MÁS PRODUCTIVOS EN EL ÁMBITO DE LOS } \\
AGRONEGOCIOS DE 2001 A 2020 \\
\begin{tabular}{|l|c|}
\hline \multicolumn{1}{|c|}{ Autor (es) } & Publicaciones \\
\hline Guilhoto, J.J.M. & 3 \\
\hline $\begin{array}{l}\text { Baggio, D.K.; Carvalho, M.M. ; Ceroni, M.; Gzain, M.; } \\
\text { Hernández, V.; Monteiro, M.d.S.L.; Montoya, M.A.; Pesce, } \\
\text { G.; Schmidt, C.M.; Schmidt, M.A.; Scoponi,; } \\
\text { Wenningkamp, K.R. }\end{array}$ & 24 \\
Fuente: Elaboración propia. & \\
\hline
\end{tabular}
\end{tabular}

En línea con lo anterior, la tabla 3 agrupa las publicaciones con base a las organizaciones que patrocinan o representan a los investigadores, en este sentido, las más productivas con relación a los agronegocios son: Association of Professionals and Technicians of CONICIT (Venezuela) y la University Center of Maringa - CESUMAR Brazilian Society of Rural Economy and Sociology (Brasil). 


\section{FIGURA 3. NUBE DE LOS AUTORES MÁS CITADOS EN EL ÁREA DE LOS} AGRONEGOCIOS DE 2001 A 2020

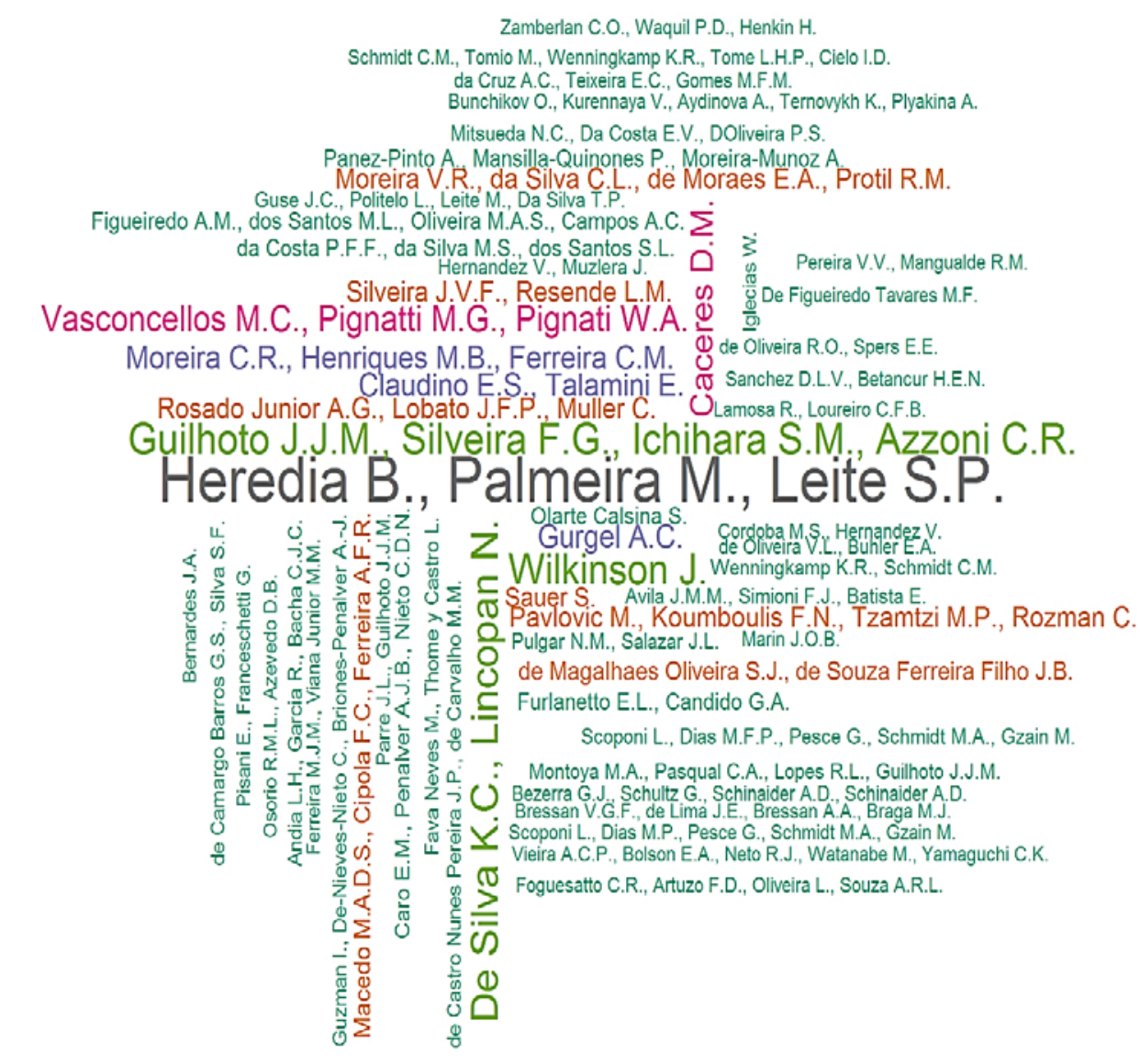

Fuente: Elaboración propia

TABLA 3. ORGANIZACIONES MÁS PRODUCTIVAS EN EL ÁMBITO DE LOS AGRONEGOCIOS DE 2001 A 2020

\begin{tabular}{|l|c|}
\hline \multicolumn{1}{|c|}{ Organización } & Publicaciones \\
\hline Association of Professionals and Technicians of CONICIT & 24 \\
\hline University Centre of Maringa - CESUMAR & 14 \\
\hline $\begin{array}{l}\text { Brazilian Society of Rural Economy and Sociology - } \\
\text { CESUMAR }\end{array}$ & 11 \\
\hline Universidad Nacional de La Plata & 6 \\
\hline Universidad de los Andes & 5 \\
\hline SAGE Publications & 3 \\
\hline
\end{tabular}

Fuente: Elaboración propia. 


\section{ANÁLISIS DE PUBLICACIONES MÁS CITADAS ÍNDICE H}

En términos de publicación más relevantes, según el índice-h de 8 que ofrece Scopus (al menos han sido citados 8 veces como mínimo), se identifican ocho publicaciones como altamente citadas. Así mismo en la Figura 1 también se puede analizar que a partir del 2012 tiene una relación directa con la cantidad de publicaciones y una tendencia creciente constante hasta el 2019. En la tabla 4 se presentan los 8 autores más representativos según el índice-h son: Heredia B., Palmeira M., y Leite S.P. con una participación en citas del 24.75 por cien, y una (1) publicación, le sigue Guilhoto J.J.M., Silveira F.G., Ichihara S.M., Azzoni C.R. con 14.29 por cien, siendo Guilhoto participante de 3 publicaciones en el ámbito de los agronegocios.

Así mismo las organizaciones más representativas son la Universidad de Sao Paulo - USP con dos publicaciones, seguido por UFGD, Research and Development Center of Peixes Ornamentais, National University of Cordoba, Federal University of Mato Grosso, Federal University of Rio Grande do Sul, Federal Rural University of Rio de Janeiro, State University of Campinas, Saude Institute of Sao Paulo y Fishing Institute. Se debe tener presente que estas organizaciones no se encuentran entre las más productivas en la sección anterior ya que son las referentes del índice-h que reflejan una diferencia entre la productividad y la representatividad en citas.

Las principales áreas de investigación relacionadas con los agronegocios entre 2001 y 2020 y las que coinciden con la anterior sección son Agricultural and Biological Sciences, Social Sciences y Economics, Econometrics and Finance. Por otro lado, las fuentes que también coinciden con la sección anterior son Revista Brasileira de Engenharia Agrícola e Ambiental y la Revista Brasileira de Zootecnia.

\section{TABLA 4. AUTORES MÁS REPRESENTATIVOS SEGÚN EL ÍNDICE-H EN EL ÁMBITO DE LOS AGRONEGOCIOS DE 2001 A 2020}

\begin{tabular}{|l|c|c|}
\hline \multicolumn{1}{|c|}{ Autores } & Citas & Citas (\%) \\
\hline Heredia B., Palmeira M., Leite S.P. & 26 & $24.76 \%$ \\
\hline Guilhoto J.J.M., Silveira F.G., Ichihara S.M., Azzoni C.R. & 15 & $14.29 \%$ \\
\hline De Silva K.C., Lincopan N. & 14 & $13.33 \%$ \\
\hline Wilkinson J. & 14 & $13.33 \%$ \\
\hline Caceres D.M. & 10 & $9.52 \%$ \\
\hline Vasconcellos M.C., Pignatti M.G., Pignati W.A. & 10 & $9.52 \%$ \\
\hline Moreira C.R., Henriques M.B., Ferreira C.M. & 8 & $7.62 \%$ \\
\hline Claudino E.S., Talamini E. & 8 & $7.62 \%$ \\
\hline
\end{tabular}

Fuente: Elaboración propia.

\section{ANÁLISIS DEL MAPA DE EVOLUCIÓN DE LOS AGRONEGOCIOS}

La evolución de los agronegocios se evaluó con base en los temas de investigación más representativos, se han propuesto dos mapas científicos, utilizando como eje los documentos extraídos de Scopus y procesados con VOSviewer. (Otegi-Olaso, y LópezRobles, 2018). VOSviewer es un software que permite construir redes bibliométricas, así como visualizar las relaciones que hay entre los temas de investigación interpretando así, la relación que hay entre las mismas. Además, es importante mencionar que el tamaño de los círculos representa la cantidad de documentos que hay sobre ese tema, y el grosor de 
las líneas representa el número de documentos que comparten. (López-Robles et al., 2020).

En la Figura 4, se encuentran las 46 líneas de investigación que forman tres agrupaciones de diferente color, las líneas muestran la intensidad de la vinculación entre cada una, con base en las similitudes de contenido en la misma publicación (LópezRobles et al., 2018). Por ejemplo, las líneas de investigación con color rojo están estrechamente relacionadas con el ámbito de los agronegocios (agribusiness), aun así, también se guardan vínculos con otras líneas de investigación de otras agrupaciones ya que es un solo ámbito de investigación, así mismo, el grosor de la línea muestra la conexión entre dos líneas de investigación que indican la fuerza que hay entre ambas, por ejemplo, agribusiness y political agronomy.

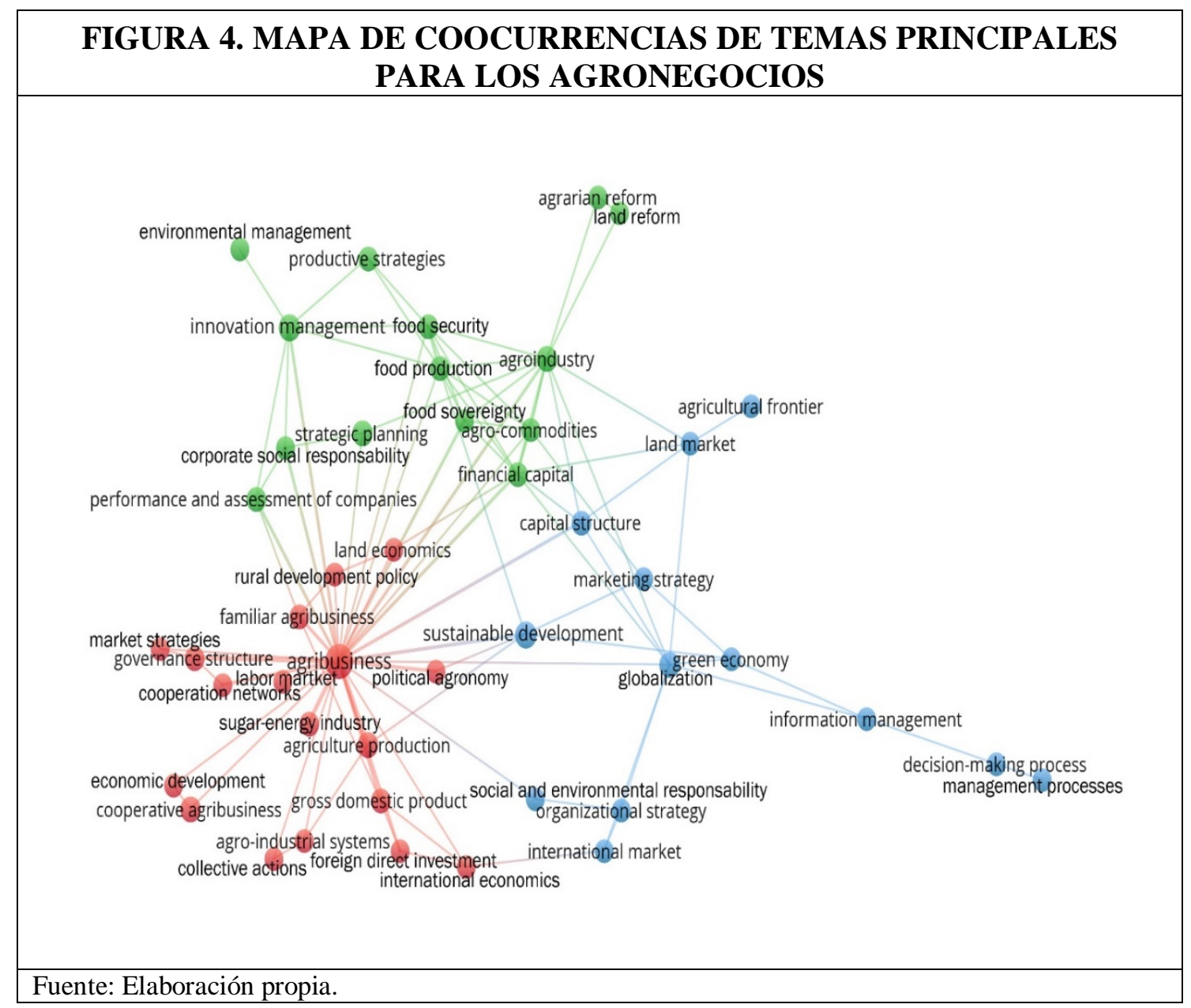

En la tabla 5 se presentan las 46 líneas de investigación estructuradas con un total 174 ocurrencias y una fuerza de vinculación de 244. Con base al número de ocurrencias y la vinculación se identifican tres agrupaciones representadas por agribusiness, innovation management y sustainable development respectivamente, la agrupación 1 suma el 55.73 por cien de las ocurrencias seguido por la agrupación 2 con 25.27 por cien, y por último la agrupación 3 con el 18.97 por cien.

La agrupación 1 aglutina las líneas de investigación que soportan directamente los agronegocios y hacen de estos una parte fundamental de la economía, mientras que la agrupación 2 centra su importancia en la innovación, es decir, agrupa las líneas de 
investigación que buscan incorporar nuevas técnicas y procesos para hacer de los agronegocios algo que sea legal, comercial, ambiental y socialmente aceptado. Por último, la agrupación 3, identifica las líneas de investigación que proyectan los agronegocios hacia la globalización y desarrollo sostenible incorporando nuevas estrategias y participando en las políticas públicas y de mercado.

Por último, en la figura 5 se presenta el mapa científico de evolución donde se refleja la evolución de los agronegocios es sus inicios vemos information management, gross domestic, el fortalecimiento del término agribusiness se ve reflejado en el 2014, las actuales tendencias en agronegocios, en una escala de tiempo son: innovation managment, corporate social responsability, y management process, agraria reform, agricultural fronteir, enviromental management, food security,collective actions, agroindustrial systems, cooperative agribusiness y strategic planning.

\begin{tabular}{|c|c|c|c|c|}
\hline \multicolumn{5}{|c|}{$\begin{array}{l}\text { TABLA 5. PRINCIPALES LÍNEAS DE INVESTIGACIÓN PARA EL } \\
\text { ÁMBITO DE LOS AGRONEGOCIO }\end{array}$} \\
\hline Línea de investigación & Ocurrencias & $\begin{array}{c}\% \text { Total } \\
\text { Ocurrencias }\end{array}$ & Vínculos & $\begin{array}{l}\text { \% Total de } \\
\text { Vínculos }\end{array}$ \\
\hline Agribusiness & 54 & $31.03 \%$ & 51 & $20.90 \%$ \\
\hline Innovation management & 6 & $3.45 \%$ & 8 & $3.28 \%$ \\
\hline Sustainable development & 6 & $3.45 \%$ & 7 & $2.87 \%$ \\
\hline Agriculture production & 5 & $2.87 \%$ & 6 & $2.46 \%$ \\
\hline Agroindustry & 5 & $2.87 \%$ & 14 & $5.74 \%$ \\
\hline Cooperative agribusiness & 4 & $2.30 \%$ & 2 & $0.82 \%$ \\
\hline Globalization & 4 & $2.30 \%$ & 9 & $3.69 \%$ \\
\hline Strategic planning & 4 & $2.30 \%$ & 3 & $1.23 \%$ \\
\hline Capital structure & 3 & $1.72 \%$ & 6 & $2.46 \%$ \\
\hline Familiar agribusiness & 3 & $1.72 \%$ & 5 & $2.05 \%$ \\
\hline Financial capital & 3 & $1.72 \%$ & 12 & $4.92 \%$ \\
\hline Food production & 3 & $1.72 \%$ & 10 & $4.10 \%$ \\
\hline Food security & 3 & $1.72 \%$ & 9 & $3.69 \%$ \\
\hline governance structure & 3 & $1.72 \%$ & 5 & $2.05 \%$ \\
\hline Gross domestic & 3 & $1.72 \%$ & 5 & $2.05 \%$ \\
\hline $\begin{array}{l}\text { Performance and } \\
\text { assessment of companies }\end{array}$ & 3 & $1.72 \%$ & 5 & $2.05 \%$ \\
\hline Productive strategies & 3 & $1.72 \%$ & 3 & $1.23 \%$ \\
\hline Sugar-energy industry & 3 & $1.72 \%$ & 3 & $1.23 \%$ \\
\hline Agrarian reform & 2 & $1.15 \%$ & 2 & $0.82 \%$ \\
\hline agricultural frontier & 2 & $1.15 \%$ & 1 & $0.41 \%$ \\
\hline Agro-commodities & 2 & $1.15 \%$ & 7 & $2.87 \%$ \\
\hline Agro-industrial systems & 2 & $1.15 \%$ & 3 & $1.23 \%$ \\
\hline Collective actions & 2 & $1.15 \%$ & 2 & $0.82 \%$ \\
\hline Cooperation networks & 2 & $1.15 \%$ & 3 & $1.23 \%$ \\
\hline \begin{tabular}{|l|} 
Corporate social \\
responsibility
\end{tabular} & 2 & $1.15 \%$ & 5 & $2.05 \%$ \\
\hline
\end{tabular}




\begin{tabular}{|l|c|c|c|c|} 
Decision-making process & 2 & $1.15 \%$ & 2 & $0.82 \%$ \\
\hline Economic development & 2 & $1.15 \%$ & 2 & $0.82 \%$ \\
\hline Economic performance & 2 & $1.15 \%$ & 0 & $0.00 \%$ \\
\hline $\begin{array}{l}\text { Environmental } \\
\text { management }\end{array}$ & 2 & $1.15 \%$ & 1 & $0.41 \%$ \\
\hline Food sovereignty & 2 & $1.15 \%$ & 7 & $2.87 \%$ \\
\hline Foreign direct investment & 2 & $1.15 \%$ & 4 & $1.64 \%$ \\
\hline Green economy & 2 & $1.15 \%$ & 4 & $1.64 \%$ \\
\hline Information management & 2 & $1.15 \%$ & 3 & $1.23 \%$ \\
\hline International economics & 2 & $1.15 \%$ & 4 & $1.64 \%$ \\
\hline International market & 2 & $1.15 \%$ & 3 & $1.23 \%$ \\
\hline Labor market & 2 & $1.15 \%$ & 1 & $0.41 \%$ \\
\hline Land economics & 2 & $1.15 \%$ & 3 & $1.23 \%$ \\
\hline Land market & 2 & $1.15 \%$ & 5 & $2.05 \%$ \\
\hline Land reform & 2 & $1.15 \%$ & 2 & $0.82 \%$ \\
\hline Management processes & 2 & $1.15 \%$ & 1 & $0.41 \%$ \\
\hline Market strategies & 2 & $1.15 \%$ & 2 & $0.82 \%$ \\
\hline Marketing strategy & 2 & $1.15 \%$ & 3 & $1.23 \%$ \\
\hline Organizational strategy & 2 & $1.15 \%$ & 3 & $1.23 \%$ \\
\hline Political agronomy & 2 & $1.15 \%$ & 3 & $1.23 \%$ \\
\hline Rural development policy & 2 & $1.15 \%$ & 3 & $1.23 \%$ \\
\hline $\begin{array}{l}\text { Social and environmental } \\
\text { responsibility }\end{array}$ & 2 & $1.15 \%$ & 2 & $0.82 \%$ \\
\hline
\end{tabular}

Fuente: Elaboración propia.

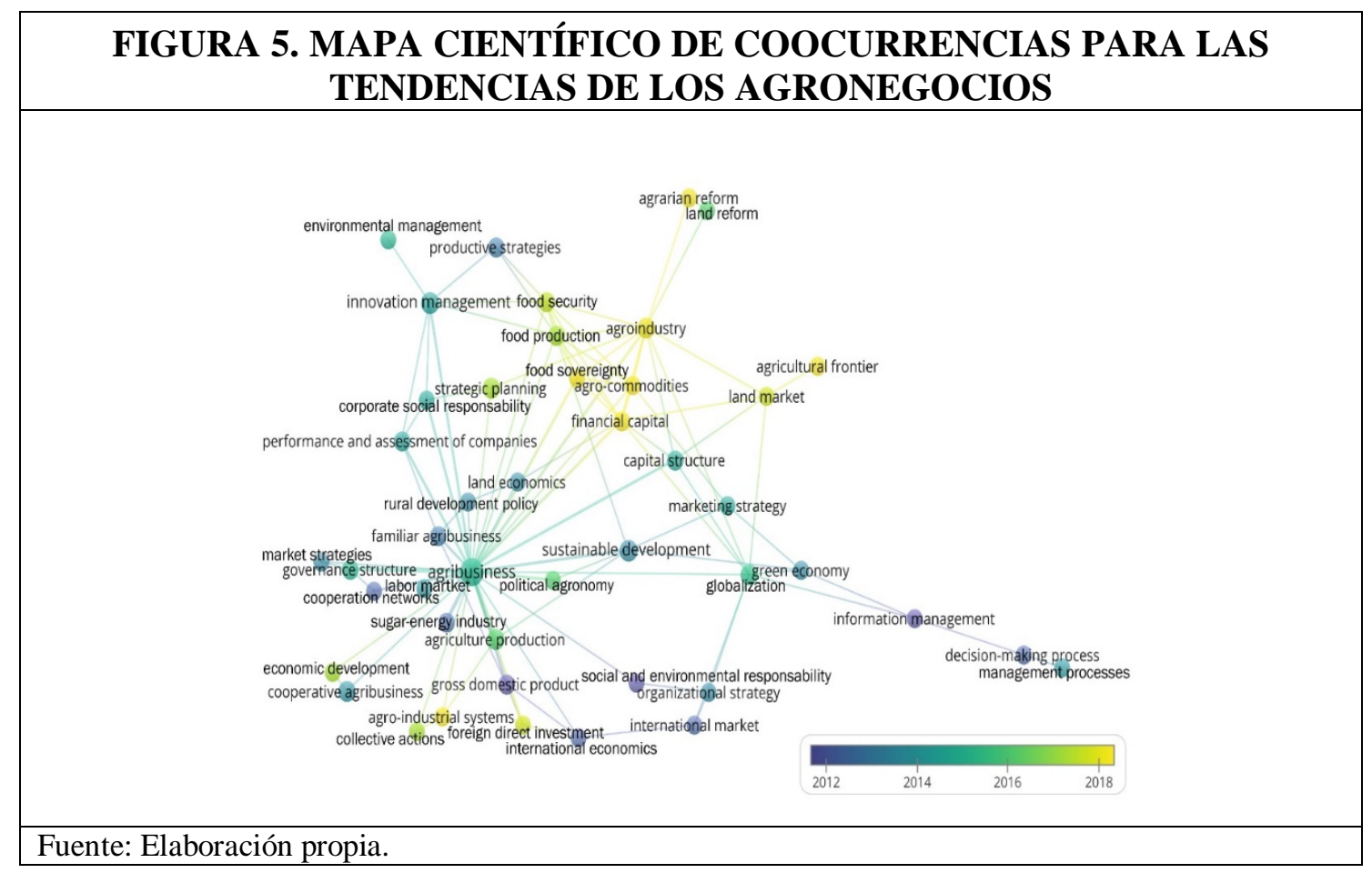




\section{CONCLUSIONES}

Con base en el análisis bibliométrico de los 121 documentos relacionados con agronegocios recuperados de Scopus, su evolución en el entorno académico muestra una perspectiva integral y representativa de los principales enfoques de estudio durante el periodo de 2001 a 2020.

En las últimas dos décadas se ha presentado un aumento de las publicaciones relacionadas con agronegocios, agrupando la mayor parte durante el periodo 2015-2018, relacionado con el auge del estudio de estrategias y prácticas para la optimización de las técnicas utilizadas en las operaciones.

La tendencia indica que sigue en aumento el número de publicaciones y citas por académicos y profesionales que profundizan en el estudio, análisis e influencia de los agronegocios y de las áreas de conocimiento relevantes identificadas en el mapa de coocurrencias.

Las fuentes de información y países más productivos en el campo de agronegocios, se concentran en Venezuela y Brasil, destacando las revistas; Espacios , Revista en Agronegocio e Meio Ambiente y Revista de Economía y Sociología Rural, agrupándose en tres principales áreas de conocimiento: Business and International Management, Agricultural and Biological Sciences (miscellaneous) y Social Sciences (miscellaneous), que a su vez son auspiciadas por organizaciones como la Asociación de Profesionales y Técnicos del CONICIT, University Centre of Maringa - CESUMAR y Sociedade Brasileira de Economía e Sociología Rural.

Otro factor a tener en cuenta, es la producción y citas de las publicaciones. Por un lado, se tiene los autores más productivos, aquellos que han sido más representativos en cuanto al volumen de sus trabajos académicos, que suscitan la evolución del desarrollo de los agronegocios en el campo científico. Estas acciones promueven nuevos enfoques de estudio en el área, siendo el autor más productivo, Guilhoto, J.J.M. Además, los autores más citados son Heredia B., Palmeira M., Leite S.P. y Guilhoto J.J.M., Silveira F.G., Ichihara S.M. y Azzoni C.R.

En lo que refiere al mapa científico, se puede identificar los principales enfoques de materia de estudio, lo cual representa las líneas de investigación más utilizadas en las publicaciones recopiladas (López-Robles et al., 2019). En este sentido, se observan las relaciones entre las mismas y su relevancia a través de los años, resaltando tres aspectos de las cuales se estima su progreso hacia la orientación académica, científica y organizacional en los próximos años, siendo las más relevantes:

- Planeación estratégica (collective actions, agroindustrial systems, cooperative agribusiness y strategic planning). Los agronegocios como todo sector productivo, se desarrollan en un entorno altamente competitivo y globalizado, por lo que los mercados cambiantes han reconfigurado las estructuras organizacionales de los agronegocios, buscando una significante apertura en la economía y los mercados, suscitando nuevas oportunidades, por las cuales se regenera por medio del establecimiento de metas organizacionales. Sin embargo, para el crecimiento de los productores y el sector; es importante establecer las acciones colectivas, la cooperación empresarial y la estructuración de nuevas técnicas de optimización agropecuarias, para tener un mayor impacto y posicionamiento en los mercados objetivos.

- Gestión de regulaciones y normatividad (agraría reform, agricultural fronteir, enviromental management y food security). Para asegurar una mayor productividad de los agronegocios y un crecimiento económico de los sectores y actores involucrados, es 
indispensable que cada país desarrolle e implemente políticas agrarias orientadas a la sostenibilidad: económica, social, institucional, ambiental y fiscal. Estas políticas deben promover el fomento de beneficios económicos reales, el bienestar de productores de pequeña y mediana escala, programas de financiamiento y asesoramiento técnico, y el desarrollo de la bioeconomía por medio de productos y servicios diferenciales.

- Gestión de innovación y tecnología (innovation managment, corporate social responsability, y management process). La innovación y la responsabilidad social influyen de manera positiva en el ámbito de los agronegocios, su aplicación en todos los procesos de la cadena valor por medio del intercambio de conocimientos e información de los agentes involucrados. Estas relaciones permiten un sistema más compacto para la aplicación de estrategias, manteniendo al consumidor final como foco principal de todas las operaciones realizadas, buscando generar buenas prácticas agrícolas para un entorno más sostenible.

Por último, los resultados obtenidos, permiten visualizar los principales enfoques de investigación relacionados con los agronegocios, enfatizando la importancia de la literatura en este ámbito en los últimos años. Esto permite que tanto investigadores como profesionales de las áreas administrativas, naturales y sociales puedan tener una perspectiva más amplia de las principales líneas de investigación y enfoques de los agronegocios. Asimismo, se direcciona la información para facilitar la creación de nuevos enfoques de estudio, y dar como resultado mayor intercambio de información y conocimiento en este campo, a través de las publicaciones científicas.

\section{BIBLIOGRAFÍA}

Arbeletche, P. y Gutiérrez, G. (2010): “Crecimiento de la agricultura en Uruguay: exclusión social o integración económica en redes”. Pampa. Revista Interuniversitaria de Estudios Terri-toriales, ${ }^{\circ}$ 6, pp. 113-138.

Bordons M, Fernández MT, Gómez I. (2002): “Advantages and limitations in the use of impact factor measures for the assessment of research performance in a peripheral country”, Scientometrics, vol. 53, n 2, pp. 195-206.

Borlaug, N.E. (2000): "Ending world hunger. The promise of biotechnology and the threat of antiscience zealotry”. Plant Physiology, vol. 124, n² 2, pp 487-490.

Börner, K., Chen, C., \& Boyack, K. W. (2003): "Visualizing knowledge domains". Annual Review of Information Science and Technology, vol. 37, $\mathrm{n}^{\circ} 1$, pp. 179255. https://doi.org/10.1002/aris.1440370106.

Buter RK, Noyons ECM, Mackelenbergh M. van y Laine T. (2006): “Combinando mapas conceptuales y mapas bibliométricos: Primeras exploraciones”, Scientometrics, vol. 66, n ${ }^{\circ}$ 2, pp. 377-387.

Cáceres, Daniel M (2015): "Tecnología agropecuaria y agronegocios. La lógica subyacente del modelo tecnológico dominante", Mundo Agrario, vol. 16.

Cáceres, Daniel M. (2015): “Tecnología agropecuaria y agronegocios. La lógica subyacente del modelo tecnológico dominante”, Mundo Agrario, vol. 16.

Castellanos, E. R. (2013): "La competitividad de los agronegocios en Colombia: una reflexión académica” Magazín Empresarial, vol. 9, n² 22, pp. 29-34.

CEPAL. (2018): La Agenda 2030 y los Objetivos de Desarrollo Sostenible Una oportunidad para América Latina y el Caribe. https://repositorio.cepal.org/bitstream/handle/11362/40155/24/S1801141_es.pdf 
Cerón-Muñoz, Mario \& Barrios, Dursun. (2019): “Agricultura de precisión: una contribución a la gestión de los agronegocios desde la modelación”, Revista Colombiana de Ciencias Pecuarias. $\mathrm{n}^{\circ} 32$.

Cilloniz, F.; Grozo, José; Riva, Luigi; Guzmán, Ángel (2003): Cadenas productivas y desarrollo empresarial. Edit. Banco Interamericano de Desarrollo; Perú; 61 pp.

Cobo, M. J., López-Herrera, A. G., Herrera-Viedma, E., \& Herrera, F. (2011): "Science mapping software tools: Review, analysis, and cooperative study among tools". Journal of the American Society for Information Science and Technology, vol. 62, $\mathrm{n}^{\mathrm{o}}$ 7, pp. 1382-1402. https://doi.org/10.1002/asi.21525.

D. Rodríguez. [coord.]. (2010): Desarrollo de los agronegocios y la agroindustria rural en América Latina y el Caribe: conceptos, instrumentos y casos de cooperación técnica. San José, Costa Rica: IIC

Garfield, E. (1977): "Introducing citation classics. The human side of scientific reports”, Current Comments, vol. 1, n ${ }^{0}$ 1, pp. 5-7.

Gras, C. y Hernández, V. (2013): El agro como negocio. Producción, sociedad y territorios en la globalización, Biblos.

Griffin, K. (1982). La economía política del cambio agrario. México: FCE

Guzmán, S. M. Trujillo, J. (2013): "Los mapas bibliométricos o mapas de la ciencia: una herramienta útil para desarrollar estudios métricos de información”. Revista Unam. Biblioteca Universitaria [en linea]. vol. 16, nº 2, pp. 95-108. ISSN: 0187$750 X$.

Heredia, B., Palmeira, M. \& Leite, S. (2010): "Sociedade e economia do "Agronegócio" no Brasil.” Revista Brasileira de Ciéncias Sociais, vol. 25, nº 74, pp. 159- 196. En http://www.scielo.br/pdf/rbcsoc/v25n74/a10v2574.pdf.

Hirsch, J. E. (2005). “An index to quantify an individual's scientific research output”. Proceedings of the National Academy of Sciences, vol. 102, n 46, pp. 1656916572. https://doi.org/10.1073/pnas.0507655102.

Ihaka, R. y Gentleman, R. (1996). "R: A language for data analysis andgraphics”, Journal of Computational and Graphical Statistics, $\mathrm{n}^{\circ}$ 5, pp. 299-314. doi: 10.1080/ 10618600.1996.10474713.

John H. Davis and Ray A. Goldberg. Boston (1957): A Concept of Agribusiness, Division of Research, Graduate School of Business Administration, Harvard University. Pp. xiv, 136.

Kalman, Judith y Rendón, Víctor (2016): “Uso de la hoja de cálculo para analizar datos cualitativos” Magis. Revista Internacional de Investigación en Educación. Vol. 9, $\mathrm{n}^{\circ}$ 18, pp. 29-48. [fecha de Consulta 13 de Abril de 2020]. ISSN: 2027-1174. Disponible en: https://www.redalyc.org/articulo.oa?id=2810/281049122003.

Leff, E. (1994): Ecología y capital: racionalidad ambiental, democracia participativa y desarrollo sustentable. México: Siglo XXI.

López-Robles, J. R., Otegi-Olaso, J. R., \& Porto-Gómez, I. (2018): Bibliometric analysis of worldwide scientific literature in Project Management Techniques and Tools over the past 50 years: 1967-2017.

López-Robles, J. R., Otegi-Olaso, J. R., Gamboa-Rosales, N. K., Gamboa-Rosales, H., Robles-Berumen, H., \& Gamboa-Rosales, A. (2019): Visualizing and mapping the project management research areas within the International Journal of Project Management: A bibliometric analysis from 1983 to 2018.

López-Robles, J. R., Otegi-Olaso, J. R., Porto-Gómez, I., Gamboa-Rosales, H., y Gamboa-Rosales, N. K. (2020): La relación entre Inteligencia de Negocio e Inteligencia Competitiva: un análisis retrospectivo y bibliométrico de la literatura 
de 1959 a 2017. Revista Española de Documentación Científica, vol. 43, n 1, pp. 256.

López-Robles, J. R., Otegi-Olaso, J. R., Porto-Gómez, I., Gamboa-Rosales, H., y Gamboa-Rosales, N. K. (2018): Inteligencia: origen, evolución y tendencias (Intelligence: origin, evolution and trends). VISIO 2018 Conference.

M. Ceroni,(2018): "Rasgos centrales del agronegocio en Latinoamérica: la experiencia en Uruguay Perfiles Latinoamericanos”, Flacso México, vol. 26, nº 52, | doi: 10.18504/pl2652-004-2018.

Martínez Castillo, R. (2010): “Soberanía agroalimentaria: características, obstáculos y perspectivas", Ciencia y Sociedad, vol. 35, n 4, pp. 623-656.

Martínez, M. A., Herrera, M., López-Gijón, J., \& Herrera-Viedma, E. (2014): "HClassics: Characterizing the concept of citation classics through H-index", Scientometrics, vol. 98, n ${ }^{\circ}$ 3, pp. 1971-1983. https://doi.org/10.1007/s11192-0131155-9.

Miguel, Sandra; Caprile, Lorena; Jorquera-Vidal, Israel. (2008): “Análisis de co-términos y de redes sociales para la generación de mapas temáticos”. En: El profesional de la información. vol. 17, $\mathrm{n}^{\circ}$ 6, pp. 637-646.

Otegi-Olaso, J. R., y López-Robles, J. R. (2018): Las competencias profesionales en las revistas científicas de Dirección de Proyectos. XXII Congreso Internacional de Dirección e Ingeniería de Proyectos.

Patel, R. (2012): "The long green revolution." Journal of Peasant Studies, vol. 40, n 1 , pp. 1-63.

Pingali, P.L. (2012): "Green Revolution: impacts, limits, and the path ahead", Proceedings of the National Academy of Sciences of the United States of America, vol. 109, $\mathrm{n}^{\mathrm{o}}$ 31, pp. 12302-12308.

Posada, M.; Martínez De Ibarreta, M. y Pucciarelli, P. (1996): "Agroindustria y cambio tecnológico. Elementos para su análisis en América latina", Problemas del Desarrollo, vol. 27, $\mathrm{n}^{\mathrm{o}}$ 105, pp. 81-105.

Rojas, P. y Sepúlveda, S. (1999): ¿Qué es la competitividad? San José, Costa Rica: IICA Teubal, Miguel (2005). “Globalización y nueva ruralidad en América Latina”, en: Norma Giarraca (Comp.), ¿Una nueva ruralidad para América Latina?, pp. 47-69, CLACSO, Argentina

Tippmann, S. (2015): "Programming tools: Adventures with R", Nature, 109-110. doi:10.1038/517109a.

Van Eck N.J., Waltman L.(2009): "Software survey: VOSviewer, a computer program for bibliometric mapping" Scientometrics. 2010; $\mathrm{n}^{\mathrm{o}}$ 84, pp. 523-538. doi: 10.1007/s11192-009-0146-3.

Van Eck, N.J.; Waltman, L. (2009): "How to normalize co-occurrence data? An analysis of some well-known similarity measures", Journal of the Association for Information Science and Technology, vol.60, $\mathrm{n}^{\circ}$ 8, pp.1635-1651. 